\title{
Energy Spectrum CT Differential Diagnosis of Idiopathic Calcification and Ossification of Adult Intervertebral Disc: A Report of three Cases
}

\section{Xiaolin Wu}

affiliated hospital of qingdao university

Hongfei Xiang

affiliated hospital of qingdao university

Wenbin Cong

affiliated hospital of qingdao university

Guoqing Zhang

affiliated hospital of qingdao university

bohua chen ( $\boldsymbol{\sigma}$ bhchen@hotmail.com )

affiliated hospital of qingdao university

\section{Case report}

Keywords: intervertebral disc, idiopathic calcification, ossification, energy spectrum CT, energy spectrum diagnosis

Posted Date: August 31st, 2020

DOI: https://doi.org/10.21203/rs.3.rs-64325/v2

License: (c) (1) This work is licensed under a Creative Commons Attribution 4.0 International License.

Read Full License 
The authors have withdrawn this preprint from Research Square 